\title{
Towards the Manufacture of Megakaryocytes and Platelets for Clinical Application
}

\author{
Anja Baigger Rainer Blasczyk Constanca Figueiredo \\ Institute for Transfusion Medicine, Hanover Medical School, Hanover, Germany
}

\section{Keywords}

In vitro thrombopoiesis - (Trans-)Differentiation . Bioreactor · Platelet transfusion · Drug carrier

\section{Summary}

Platelet transfusions are used in standard clinical practice to prevent hemorrhage in patients suffering from thrombocytopenia or platelet dysfunctions. Recently, a constant rise on the demand of platelets for transfusion has been registered. This may be associated with several factors including demographic changes, population aging as well as incidence and prevalence of hematological diseases. In addition, platelet-regenerative properties have been started to be exploited in different areas such as tissue remodeling and anti-cancer therapies. These new applications are also expected to increase the future demand on platelets. Thus, in vitro generated platelets may constitute a highly desirable alternative to meet the rising demand on platelets. Several factors have been considered in the road trip of producing in vitro megakaryocytes and platelets for clinical application. From selection of the cell source, differentiation protocols and culture conditions to the design of optimal bioreactors, several strategies have been proposed to maximize production yields while preserving functionality. This review summarizes new advances in megakaryocyte and platelet differentiation and their production upscaling.

(C) 2017 S. Karger GmbH, Freiburg

\section{Introduction}

Platelets (PLTs) fulfill essential functions in primary hemostasis and wound healing [1] and maintain immunological properties [2, 3 ], but also play a role in inflammation and cancer [4]. In vivo, up to $3 \times 10^{3}, 2-3 \mu \mathrm{m}$ small PLTs are formed by demarcation and cytoplasmatic shedding from one large precursor cell known as megakaryocyte (MK) [5]. MKs reside within the bone marrow where they differentiate from hematopoietic stem cells within the osteoblastic niche $[6,7]$. During their maturation they undergo multiple endomitoses [8], migrate to the sinusoids vessels, and extend protusions (proplatelets; proPLTs) through the vessel pores. The shear stress within the sinusoidal lumen supports the release of PLTs into the blood stream [9]. This process is tightly controlled by the microenvironment within the bone marrow vascular niche [10, 11], maintaining normal PLT levels of $150-400 \times 10^{3} \mathrm{PLTs} / \mu \mathrm{l}$ in the healthy human organism. Diverse local cell populations, including sinusoidal endothelial cells, secrete a plethora of cytokines (e.g., thrombopoietin (TPO), vascular endothelial growth factor (VEGF), interleukin 1 alpha (IL-1a) [12-14]) that regulate thrombopoiesis [15].

Understanding the basic biology of thrombopoiesis and its physiological mechanisms is fundamental to efficiently mimic PLT production in vitro [16], an approach that is gaining plenty of importance for future transfusion and regenerative medicine. The demand for PLT transfusion is constantly rising $[17,18]$. While the majority of PLT transfusions is provided to patients with reduced PLT counts after chemotherapy or hematopoietic progenitor cell transplantation [19], also other clinical causes like inherited disorders [20], immunological complications [21], infections as well as acute injury can lead to life-threatening thrombocytopenia and may require urgent therapeutic or prophylactic PLT transfusion.

Since the donor-dependent PLT supply is limited, current initiatives such as patient blood management $[22,23]$ aim to improve the appropriate use of PLTs and reduce wastage. Besides the challenge of PLT shortage, the need for sensitive handling [24], a short shelf life, problematic long-term storage [25] as well as a risk for

\section{KARGER}

(C) 2017 S. Karger GmbH, Freiburg

Fax +497614520714 
bacterial or viral contamination and the transmission of diseases [26] exacerbate the management of PLTs for transfusion. Also, multitransfused patients alloimmunized to human leukocyte or PLT antigens (HLA, HPA), show an increased risk for PLT refractoriness that cannot be completely eliminated by matching of HLA in PLT transfusions [27]. Hence, it would be desirable to overcome the need for donor PLT transfusion by establishing solid strategies for the in vitro production of designer MKs or PLTs to be applied in personalized transfusion medicine.

Furthermore, PLT-derived products have demonstrated powerful regenerative properties in the field of tissue engineering [28] or as drug carrier in anti-cancer therapies [29].

The regenerative potential of PLTs relies on the high content of growth factors like epithelial growth factor (EGF), insulin growth factor-1 (IGF-1), PLT-derived growth factor (PDGF) A and B, transforming growth factor- $\beta$ (TGF- $\beta$ ), and VEGF. In fact, PLTrich blood derivatives, such as PLT-rich plasma, PLT-rich fibrin or PLT releasate, are applied for stem cell-based tissue engineering and regeneration [30]. Moreover, the clinical use of autologous PLT products is proven in the application of PLT lysate drops to prevent graft-versus-host disease (GvHD) [31] or support wound healing in ophthalmology [32]. Hence, advantages and potential applications of PLT products are numerous. Also as supplement in cell culture for preparation of cell-based therapeutics, the use of PLT lysates gained plenty of attention [33]. In particular, the balanced interplay of multiple growth factors determines the stimulating capacity of PLT lysate [34].

Moreover, in recent years PLTs also gained therapeutic interest as drug carriers to target circulating tumor cells. PLTs are most suitable for this purpose due to their biocompatibility and degradability, their natural target release capacities and adhesive properties [35], and their affinity to cancer cells [29], especially in case of thrombocytopenia-induced tumor hemorrhage due to tumor vessel injury [36]. In addition to the need of PLTs for transfusion, these innovative applications of PLTs or their derived factors may also contribute to increase their demand. Therefore, it will be crucial to establish robust biotechnological platforms that may serve as an alternative to PLT donation and provide an adequate PLT supply to meet all needs. In the past, different studies have contributed to extensively characterize the mechanism of megakaryopoiesis and PLT production $[37,38]$. This knowledge paved the way for technical breakthroughs regarding in vitro MK and PLT production. Nevertheless, the biomanufacture of MKs and PLTs requires upscaling to achieve sufficient yields, and it has to comply with good manufacturing practice (GMP) standards to achieve clinical grade. Several attempts were developed to meet these criteria towards producing PLTs in equivalent quality standards as donor PLTs.

\section{Mimicking Megakaryopoiesis and Thrombopoiesis in vitro}

To model and reproduce physiological megakaryopoiesis and thrombopoiesis, several protocols using different cell sources have been established since the first report of in vitro generated human MKs and PLTs from CD34+ peripheral blood progenitor cells [39]. All differentiation approaches are based on TPO, which is the major key driver of thrombopoiesis [40]. TPO regulates the whole differentiation process and lately the final life span of a PLT [41].

Towards clinical application of in vitro differentiated PLTs, the developed protocols need to address special issues such as GMP feasibility, practicability including scalability to achieve clinical yields, and high PLT quality. According to the GMP for pharmaceutical products by the Food and Drug Administration (FDA), the in vitro manufacture of PLTs requires the consideration of following aspects [42]:

(1)Safety of the progenitor cell source. The medical history of the stem cell donor should be known [18] or the cell reprogramming strategy of the source for induced pluripotent stem cells (iPSCs) need to comply with GMP. In addition, reprogramming approaches would preferentially use nonintegrative vectors. Moreover, the resulting differentiation product (PLTs or MKs) has to be irradiated to reduce the risk of resting, potentially tumorigenic cells.

(2)Differentiation protocols should avoid xenogeneic components. Therefore, serum/feeder-free culture and the use of animal-free antibiotics and cytokines are recommended.

(3)Any risk of contamination should be eliminated, e.g., by the use of automated culturing systems [42].

Figure 1 summarizes some of the key factors and corresponding aspects to allow the establishment of an effective protocol for the future in vitro manufacture of MKs or PLTs.

The practicability of large-scale production of MKs and PLTs strongly relies on a robust and abundant cell source that can be easily operated and differentiated in short-time culture periods. Several genuine (e.g. CD34+ hematopoietic progenitor/stem cells, pre-adipocytes, embryonic stem cells (ESCs)) or induced (e.g. iPSCs, immortalized megakaryocytes (imMKs) and induced megakaryocytes (iMKs) from erythrocytes or fibroblasts) cell sources have been used for the in vitro production of PLTs. Although being the natural cell source for PLTs, CD34+ progenitor/stem cells are donor-dependent, available in low amounts, and show a very limited proliferative capacity. All these features remarkably restrict the feasibility to use them for the standardized large-scale production of PLTs in vitro. The use of ESCs poses significant ethical concerns which disable them as a potential cell source. Differentiation of adipose tissue-derived stem cells (ASCs) [43-45] or transdifferentiation from erythrocytes [46] and fibroblasts $[47,48]$ have been demonstrated; however, further investigations will be required prior to establishment of those cells as robust sources for PLT production. Currently, native or genetically modified iPSCs are accepted as the most promising cell source for in vitro PLT pharming due to their availability, upscalability, and low ethical concerns. Furthermore, the differentiation of a cryopreservable intermediate PLT precursor would be advantageous to onset a fast-track production of PLTs for high demands or urgent needs. The potential of using immortalized iPSC-derived MKs as direct PLT source has also been demonstrated [49] and might contribute to simplify and 


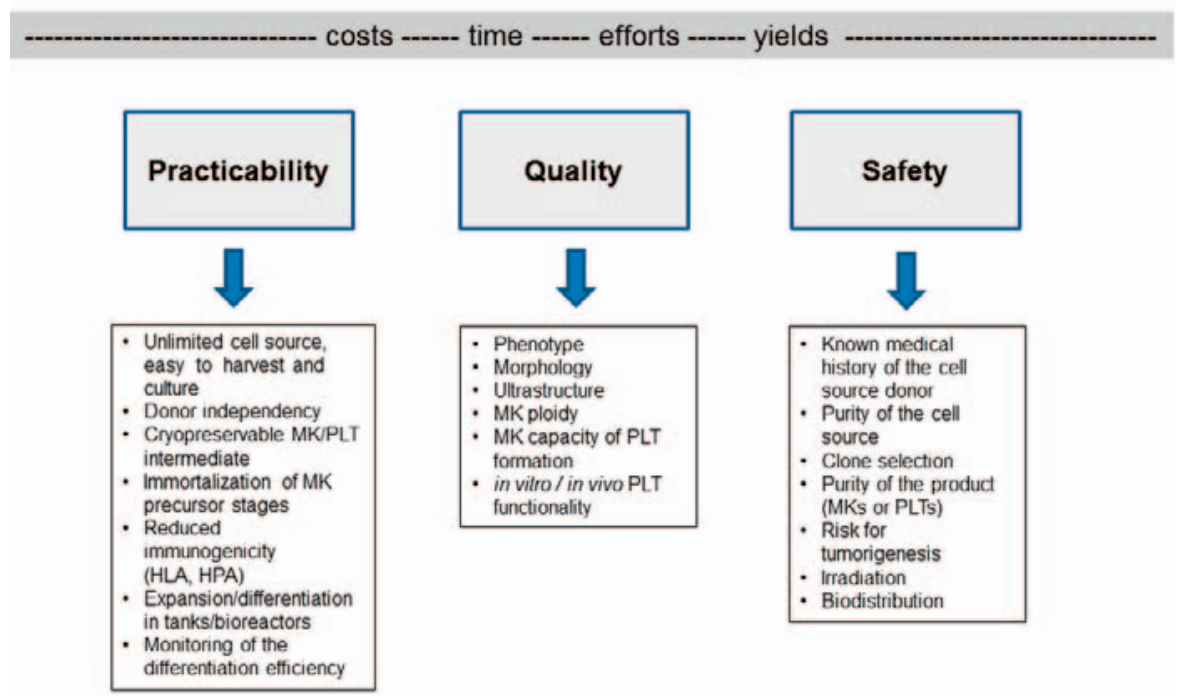

Fig. 1. To achieve safe clinical products for transfusion and regenerative medicine in sufficient yields, three building blocks for an efficient production should be considered: high practicability in production, quality tests and assurance for MKs and PLTs, as well as safety aspects. accelerate the process of PLT production. Independently of the cell source chosen for the manufacture of PLTs, all differentiation protocols should allow the culture in tanks or bioreactors to optimize and upscale the production towards clinical yield.

Generating MKs and PLTs with adequate purity (as achieved e.g. by special filter devices for PLT separation [50]) and especially appropriate quality remain major challenges for clinical application of these bioproducts. Essentially, in vitro generated MKs need to satisfy the natural characteristics of MKs derived from bone marrow [51] or cord blood [52]. Thon and colleagues [18] suggested to test in vitro MKs for their morphology, ultrastructure, cytoskeletal organization, granule content, biomarker expression, ploidy, gene expression as well as proPLT formation and PLT release. PLT quality has to be appropriate and correspond to morphological as well as functional characteristics of donor PLTs. Often, in vitro generated PLTs may differ in size and function from donor PLTs [53]. The functional characterization of in vitro PLTs may be evaluated using several innovative methodologies, as reviewed by Paniccia et al. [54]. In particular, PLTs should be analyzed for their ability to adhere and aggregate after activation and for their circulation time in macrophage-depleted animals, and for their ability to incorporate in developing mouse thrombi [18]. According to Sim et al. [16], minimal quality criteria for in vitro derived PLTs should include the responsiveness to agonists using standard aggregometry, the function in thrombus formation in vivo, and the determination of PLT half-life.

\section{Native Cell Sources}

Initially pharming of PLTs was focused on the use of donor-dependent cell sources such as CD34+ hematopoietic progenitor cells. In the early 1990s, specific cytokine cocktails supporting in vitro megakaryopoiesis were identified. Since then, several studies have described protocols to generate $\mathrm{CD} 41+\mathrm{CD} 42 \mathrm{~b}+$ polyploid megakaryocytic cells in vitro from CD34+ hematopoietic cells derived from bone marrow, umbilical cord blood, or peripheral blood
[55-57]. The differentiated MKs were capable to form proPLTs. PLTs generated in vitro according to those protocols showed to be at least partially active in vitro upon stimulation with specific agonists such as ADP, thrombin, or fibrinogen as detected by the expression of P-selectin and the active form of GPIIb-IIIa. Other studies have demonstrated the capacity of in vitro differentiated PLTs to be incorporated in thrombi in vivo [58]. Still these protocols demands further adaptation to xenofree culture conditions, and functional tests for PLTs need to be standardized.

Other donor-dependent cell sources exploited for the in vitro PLT production include stromal cells with different origins such as adipose tissue or endometrium. Ono-Uruga and colleagues [45] recently generated MKs and PLTs from human ASCs. In particular, the CD71+ subpopulation was responsive to endogenous TPO, leading to MK differentiation within 6 days, with yields of 3-15 PLTs/MK. PLTs bound fibrinogen, expressed P-selectin, and the active form of GPIIb-IIIa upon thrombin stimulation and were able to aggregate in vitro. However, PLT formation from MKs infused in a mouse model was not demonstrated.

\section{Transdifferentiation}

Sirpin et al. [46] successfully generated PLTs from MKs that have been transdifferentiated from human bone marrow erythroblasts by the overexpression of FLI1 and ERG genes. Within 7-11 days after transduction, CD41+ MKs appeared in culture. However, PLT production was dependent on induction by OP9 stromal cell culture, and aggregation was proven only in combination with donor PLTs. Furthermore, two different approaches to convert human fibroblasts to MKs have been described. In the study of Ono et al. [47], human dermal fibroblasts were transdifferentiated within about 17 days into MKs by the overexpression of $p 45 N F-E 2$, Maf $G$ and $M a f K$ genes and following culture in induction medium. The resulting CD41+ polyploid iMKs were morphologically similar to bone marrow MKs and produced CD41+ PLT-like particles upon infusion into immunodeficient mice. Moreover, the in 
vitro generated PLTs were capable of thrombus formation on a collagen-coated chip implanted in a thrombocytopenic immunodeficient mouse.

Also Pulecio et al. [48] accomplished a direct conversion of mouse and human dermal fibroblasts to CD41+ iMKs within 4-12 days by genetic engineering with GATA2, RNX1 in combination with the transcription factor core (GATA1, TAL1, LMO2, c-MYC). Each induced MKs produced 5-10 PLTs that spread on activated glass. However, some pre-activation occurred during this procedure, hence P-selectin levels did not change after challenge with $\mathrm{ADP}$ and thrombin.

\section{Pluripotent Stem Cells}

The limited availability of the cell source represents a relevant obstacle for large-scale fabrication of MKs and PLT production. Hence, the use of human embryonic stem cells (hESCs) was considered as a potential relevant cell source. hESCs can be virtually cultured indefinitely in vitro, and therefore they constitute an unlimited and donor-independent cell source. Already in 2006, Gaur et al. [59] demonstrated the feasibility to differentiate MKs from hESCs. Furthermore, hESC-derived MKs were shown to produce PLT-like particles with similar ultrastructure, morphology, and functionality when compared to blood-derived PLTs $[58,60]$.

Feng et al. [61] presented a promising approach for the differentiation of MKs and PLTs from human iPSCs via a hemogenic endothelium intermediate. In this protocol, addition of a noncytotoxic c-myc inhibitor showed to enhance MK progenitor formation. iPSC-derived PLTs were capable to aggregate in in vitro tests and were incorporated into thrombi in macrophage-depleted mice [61]. Furthermore, Liu et al. [62] achieved the differentiation of MKs from human iPSCs using FDA-approved pharmacological reagents. To reduce the risk of PLT transfusion refractoriness, iPSCs have been genetically engineered for the downregulation of HLA class I or PLT antigens using gene regulatory (RNA interference) [63] or editing (TALEN) strategies [61]. iPSC-derived HLA class I-silenced MKs and PLTs have shown to escape antibody-mediated cytotoxicity in vitro and in vivo [63]. Also, CRISPR/Cas9 gene editing of PLT antigens in iPSCs was performed [64]. These approaches aim to reduce the immunogenicity of PLT transfusions towards the generation of universal PLT products.

Other differentiation protocols that attempted the differentiation of MKs from different cell sources were based on the transient overexpression of specific transcription factors. Moreau et al. [65] described the generation of high yields of MKs by chemical forward programming (fop) of hPSCs based on the overexpression of GATA1, FLI1 and TAL1. FopMKs tolerated cryopreservation, and PLTs appeared to be functional as revealed by spreading on fibrinogen as well as in vivo thrombi formation. This approach allowed the production of means of five PTLs per CD41+ MK.

Nakamura et al. [49] established immortalized, cryopreservable MK progenitor cell lines (imMKCLs) by the differentiation of hIPSCs and hESCs that were genetically engineered to stepwise overexpress C-MYC, BMI1, and BCL-XL. This approach ensured the suppression of senescence and apoptosis while stimulating prolif- eration. Upon doxycyclin-regulated inhibition of the inducer gene,s imMKCLs produced functional CD42b+ PLTs in vitro. The authors suggest to store $10^{8} \mathrm{imMKCL}$ cells per vial as master cell bank-derived working cell stock which should produce $2.5 \times 10^{10}$ MKs in around 14 days. Also, they identified clones of imMKCLs that expanded stably in liquid culture after adaption to serum- and feeder-free conditions. However, functional discrepancies were found between in vitro derived and endogenous PLTs. According to the authors, this might be caused by the lack of serum within the in vitro system, compared to the serum-rich PLT concentrates used for transfusion. Moreover, studies in experimental transfusion models may be crucial for imMKCL clone selection, since the authors have shown that specific clones gained chromosomal translocations leading to leukemogenesis upon infusion into immunodeficient mice.

\section{Large-Scale Production of Megakaryocytes and Platelets}

To achieve clinical numbers of in vitro generated MKs and PLTs, current next-generation strategies become distanced from static cultures and open up to fluidic biomimetic reactors recapitulating the natural bone marrow environment. During the evolution of PLT bioreactor design, diverse technical and biological key features have been addressed by multiple setups (table 1), allowing the production of in vitro PLTs with characteristics, resembling donated PLTs (table 2). Selected approaches and their improvements are highlighted within the following paragraphs.

\section{The First Trials and Improvements}

In 2006 Matsungaga et al. [55] demonstrated PLT differentiation from human cord blood-derived CD34+ progenitor cells in a three-phase culture system. The first two differentiation phases were based on static cultures using hTERT human stroma cell as feeders. However, the final maturation of MKs and PLTs occurred in a six-well suspension culture system, representing a first step away from static cultures. Another new feature introduced by this study was the co-culture of MKs in combination with human umbilical vein endothelial cells (HUVEC), since endothelial cells are known to fulfil stimulatory functions on proPLT formation. Using this protocol and serum-free media, the differentiated PLTs showed a typical ultrastructure and upregulated the expression of P-selectin and activated GPIIb-IIIa upon ADP stimulation. In addition, PLTs were able to form aggregates in response to fibrinogen.

In 2009, Sullebarger et al. [66] published the first 3D PLT bioreactor built from a modular perfusion system. The device contained a central producer cell disc covered by a layer of pre-expanded CD34+ progenitor cells, while medium and gas flow occurred in separate spaces above and below this cell layer. This setup allowed the harvest of PLTs from the lower medium space over 30 days. Collected PLTs were capable to aggregate and showed surface expression of activation markers such as CD62 and CD63. Later, they 

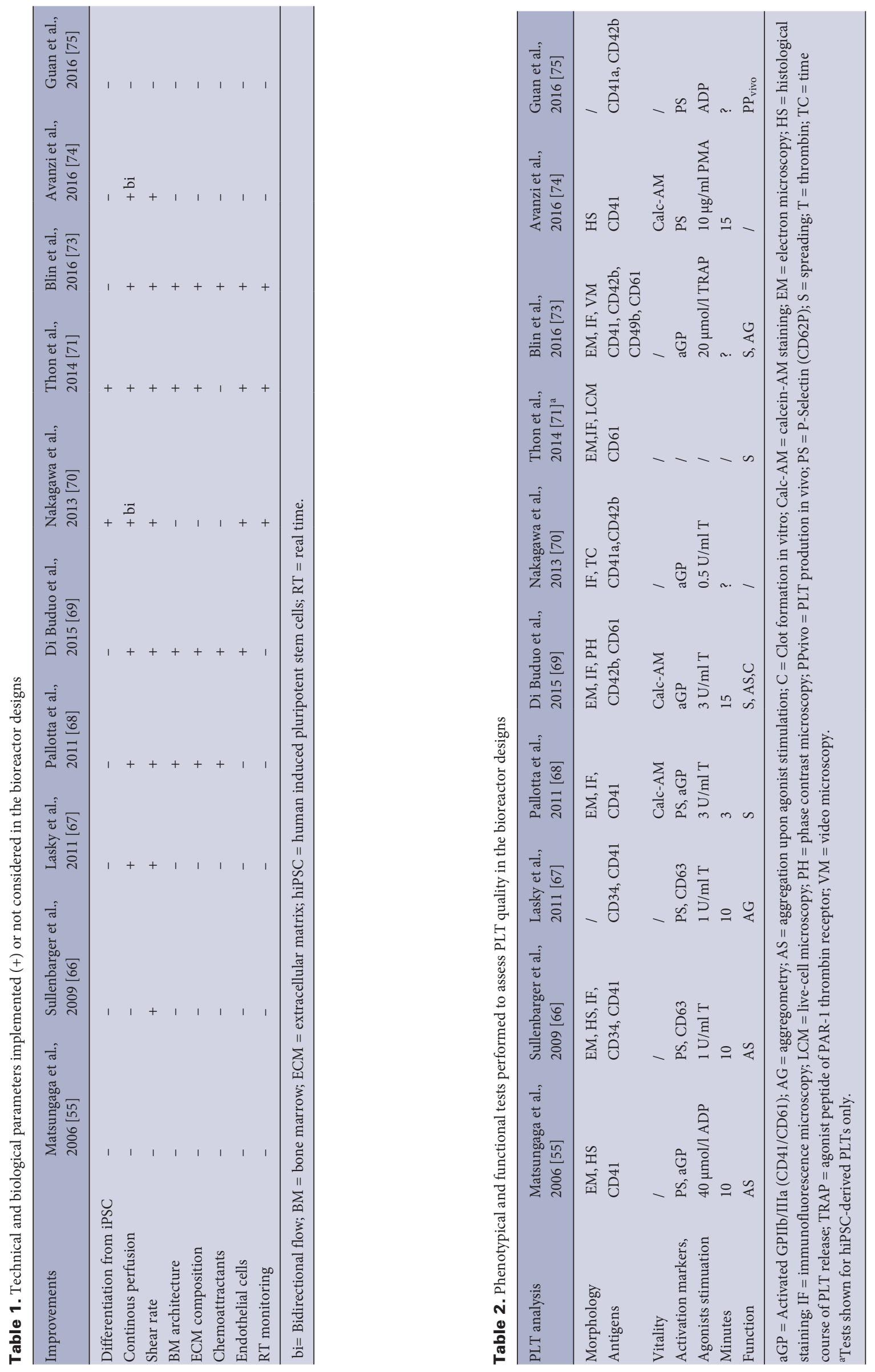
further improved this bioreactor prototype. They increased PLT production by regulating the oxygen supply and inducing controlled shear stress with help of a continuous medium flow though the cell scaffold [67]. These first approaches demonstrated the feasibility to produce PLTs not only in suspension cultures but also in continuous perfusion systems which can significantly facilitate the upscaling of PLT production.

\section{Silk-Based Bioreactor Systems}

In 2011 Pallotta et al. [68] established a 3D model one step ahead to a close technical analogue of the bone marrow microenvironment by the application of silk protein biomaterial. To simulate the natural niche, growth factor-coated silk microtubes (mimicking sinusoidal vessels) were embedded in modules filled with type I collagen gel. MKs were differentiated from CD34+ cells and seeded between the collagen gel and each microtube. Within $16 \mathrm{~h}$, they migrated towards the microtube and released proPLTs into the constitutive flow of media within the microtubes. However, only $7 \%$ of MKs exhibited proPLT production, with a yield of about 20 PLTs/MK. In 2015 Di Buduo et al. [69] presented a follow-up of this prototype, equipped with an additional silk sponge encompassing the microtube to better mimic the stiffness of the sinusoidal vessel surrounding. Moreover, they improved the entrapment of growth factors and extracellular matrix components (collagen-1, collagen-4, fibrinogen, fibronectin, type IV collagen, or laminin), and seeded HUVEC into the lumen of the silk microtubes. These new features led to a threefold increase in numbers of released PLTs. The generated PLTs demonstrated functional properties in vitro and were able to aggregate and to form thrombi. Those studies showed that natural silk protein biomaterial convinces by its biocompatibility, non-thrombogenic features, programmable mechanic properties as well as by its surface binding of cytokines, extracellular matrix components, and endothelial-derived proteins.

\section{Refined Bioreactors}

In 2013, Nakagawa et al. [70] described a next generation of technically advanced bioreactors, with the unique feature of exposing MKs to two differential flows. MKs were developed from iPSCs or hESCs using mouse feeder cells and on day 20 of the differentiation transferred to modified bioreactors. Initially MKs were seeded onto a HUVEC monolayer covering a porous PDMS membrane and exposed to a vertical flow that facilitated their migration as well as their attachment to the membrane and extension of protrusions through the membrane. A horizontal flow below the membrane allowed the shedding of PLTs from megakaryocytic proPLTs. In an improved variant of this bioreactor, MKs were settled into special slits on the membrane to expose them to the bidirectional flow in an optimized angle.

Step by step, bioreactor bioengineering for efficient PLT production became increasingly complex. In 2014, Thon et al. [71, 72] presented the first PLT bioreactor-on-a-chip that, despite its small size, considered a broad spectrum of parameters to recapitulate the bone marrow microenvironment. To mimic the stiffness of the natural bone marrow, MKs were seeded in hydrogels such as algi- nate. To improve MK trapping, extracellular matrix proteins (e.g. fibrinogen, laminin, or collagen) were added into the surrounding media, or used to coat the membrane separating the MK chamber from the lower flow chamber. ProPLT formation was stimulated with help of endothelial cell contacts, and PLT release was optimized using controlled hemodynamic vascular shear stress of $\sim 600$ Mpa. Thereby, an immediate onset of proPLT formation (completed within $2 \mathrm{~h}$ ) and an elevated percentage of proPLT-forming MKs as well as increased PLT counts could be achieved from primary mouse MKs as well as from hiPSC-derived MKs. Morphology, ultrastructure, and function of PLTs produced by hiPSC-dervied MKs corresponded to the ones of blood PLTs. Moreover, progress was made in monitoring proPLT formation and PLT release by using specialized 3D live-cell imaging microscopes that also allowed a quantification of PLT production.

\section{Latest Trends in Bioreactor Design}

In 2016 Blin et al. [73] developed a 'microfluidic model of the PLT generating organ', constituted by a single-flow chip, in which MKs derived from human cord blood (hCB) CD34+ cells were constitutively perfused and captured by thousands of $\mathrm{vWF}$ (von Willebrand factor)-coated micropillars to release PLTs into the media flow. This setup enabled a high throughput of millions of MKs within $2 \mathrm{~h}$ of perfusion using high shear rate of $1,800 / \mathrm{s}$. The collected PLTs were considered as functional according to the analysis of their microtubule dynamics and the expression of CD42b. After a series of highly specialized bioreactors such as bioreactor-on-a-chip had been developed, in the recent year more reduced, simplistic systems aiming a straight upscale of the PLT manufacture were presented. Avanzi et al. [74] introduced a culture system in combination with a novel bioreactor boosting high yields of PLTs from human cord blood-derived CD34+ progenitors. After hundredfold expansion of these CD34+ progenitors during 1 week, a MK differentiation phase was performed in serum-free media for 11 days. Subsequently CD41+ MKs were transferred to a nanofiber membrane within the bioreactor. Here, they were exposed to a bidirectional flow, causing shear stress of $30-70 / \mathrm{s}$, and stimulating the release of up to $100 \mathrm{PLTs} / \mathrm{MK}$. Also in 2016, Guan et al. [75] presented a 2-liter bottle turning device filled with $600 \mathrm{ml}$ medium for the production of MKs and PLTs from CD34+ progenitor cells. After a 6-day expansion step and initiation of the differentiation in serum-free medium, progenitor cells were transferred to the turning bottle for additional 7 days of final maturation. The MKs released PLTs with a half-life of about $10 \mathrm{~h}$ in vivo when infused into NOD/SCID mice. Moreover, MKs did not reveal altered expression levels of proto-oncogenes compared to CD34+ cells. These studies described the possibility to use simple bioreactors for in vitro PLT production. A direct comparison regarding cost, effort, PLT yields, and quality remain to be performed between simple and more complex bioreactors. Nevertheless, a precise cost-benefit analysis of the mentioned production approaches may be difficult, due to their pronounced heterogeneity and the multitude of technical features. 


\section{Clinical Application}

Due to the limited in vitro production of sufficient PLT numbers for transfusion, so far clinical studies have been mainly focused on the transfusion of in vitro generated MKs. Already in 1997 for the first time megakaryocytic progenitors differentiated in vitro from $\mathrm{CD} 34+$ cells in serum-free medium were reinfused to ten autologous recipients $\left(1-21 \times 10^{5}\right.$ in vitro generated CD61+ $\mathrm{MKs} / \mathrm{kg}$ body weight) [76]. In this study, a positive correlation between rising doses of MK progenitor infusion and the outcome of PLT recovery was observed. Some but not all patients who received highest doses of cultured MK progenitors did not require PLT transfusion support. Also in 2012, Xi et al. [77] performed a phase I clinical trial using $\mathrm{ABO}$ - and HLA-matched MK progenitors generated from cord blood mononuclear cells under serum-free conditions. A total of 24 patients with hematological malignancies were infused with a median concentration of $5.45 \times 10^{6} \mathrm{CD} 41+\mathrm{MK}$ progenitor cells/kg body weight. The MK progenitors were tolerated without severe secondary effects, and neither led to GVHD within 1 year of follow-up. PLT recovery was reached in only 12 of 19 patients who did not receive further PLT transfusion. In September 2014, the Nanfang Hospital of Southern Medical University started recruiting for a clinical study to test megakaryocytic progenitor cells for prophylaxis and treatment of thrombocytopenia [78]. Moreover, imMKCLs, produced as discussed earlier in this review [49], from voluntary blood component donors with specific HLA haplotypes are currently being tested in a clinical phase I trial in Japan, initiated by Koji Eto [79]. The group demonstrated an approach for generation of $0.5 \times 10^{11}$ imMKCL-derived PLTs from each batch of an 8-liter culture [80]. The imMKCLs are analyzed for their PLT production capacity in vivo, determining their suitability in transfusion therapy. These first results suggest the feasibility of MK transfusion as a strategy to treat thrombocytopenia. However, potential secondary loci of thrombopoiesis were observed infusing ex vivo generated human $\mathrm{MK}$ into mice, representing a potential risk for pulmonary diseases $[16,18,26]$. Nevertheless, the data generated within these clinical trials support the feasibility of using in vitro manufactured PLTs as a future alternative or complementary strategy to PLT donation.

\section{Conclusions and Outlook}

It is currently possible to efficiently differentiate MKs from iPSCs, but they show a restricted capacity to produce PLTs in vitro. Physiologically, $1 \mathrm{MK}$ produces thousands of PLTs into the circulation. In contrast, the protocols available only allow the production of up to hundreds PLTs per MK. This delays the possibility for clinical application of in vitro produced PLTs. Yields of PLT production may profit in the future from the harmonization of MK expansion and differentiation culture systems towards a synchronized PLT formation and release [74]. The application of shear stress in the designed bioreactor aimed to provide a physical cue to induce a synchronized proPLT formation, extension, and
PLT release [80]. However, it remains highly desirable to identify biological or chemical signals that might support this process. Moreover, the in vitro generated PLTs do not unfailingly suit all expected quality criteria in comparison to donor-derived PLTs [81]. Here, a standard catalogue of morphological and functional tests for the evaluation of the PLT quality in vitro and in vivo would be necessary to allow a direct comparison between the individual protocols. In vivo PLT survival and functional studies have been significantly limited due to the absence of a reliable animal model. In particular NOD/SCID mice showed to be valuable for testing survival of human PLTs in the mouse circulation after storage, exposure to human antibodies, or in pharmacological studies $[82,83]$. However, major differences between mice and human PLTs may compromise the significance of the functional data of human PLTs after infusion into the mouse circulation. Although mouse and human PTLs are similar, mouse PLTs are smaller, present in higher number in the circulation and have a different antigen expression pattern. In addition, shear stress, an elementary parameter for proPLT formation and PLT maturation, might differ in the narrow, murine vascular system that consequently might not represent the optimal environment to test production and quality of the larger human MKs or PLTs [47]. These differences might be associated with variations in responses of human and mouse PLTs to the same agent. In addition, functional assays of human PLTs in the mouse circulation are also limited by the maximal volume $(\sim 200 \mu \mathrm{l})$ possible to inject at one application and which is far away from the volumes applied in a clinical situation [82-84]. Despite the overall relevance of in vivo functional tests, studies have demonstrated the capacity of in vitro generated PLTs to adhere to the endothelium and of clotting after infusion into the mouse circulation $[47,49,58,61,65]$. These results suggest, at least in part, that in vitro generated PLTs are able to respond to stimulatory stimuli in vivo. Nevertheless, it is still necessary to establish an optimized in vivo model to investigate the fine tuning of PLT activation and function as well as interaction between PLTs with other cell types.

Furthermore, methods of collection and storage of biomanufactured PLTs have to be improved to prevent inappropriate PLT activation prior to transfusion. Despite of the use of inhibitors of PLT aggregation such as prostanglandin E1, the high susceptibility of PLTs to activation during the collection and storage process due to shear stress, centrifugation, and unsuitable composition or $\mathrm{pH}$ changes of the media remains a relevant hurdle during PLT production that may compromise their clinical efficiency [71, 85]. Thus, major efforts may be required to improve the media selected for the collection and storage of PLT produced in bioreactors.

The application of donor-independent, infinite progenitor sources for optimized PLT production such as HLA-deficient iPSCs [61, 63] or immortalized MKs [49] in close biomimetic reactors [69-71] or systems allowing a high production scalability, would enable one step further to clinical application $[74,75]$. Despite the recent advances in optimizing the features and methods for in vitro PLT production, so far the manufacture of PLTs remains ineffective, which may support the evaluation of alternative 
strategies. MK transfusion already proved in the past and in current trials to be an effective strategy to increase PLT counts in the circulation. In fact, several studies have demonstrated the feasibility of transfused MKs to produce PLTs in animal models and even in clinical studies $[76,77]$. Nevertheless, some studies have showed that in mice infused MKs might become retained in the lung vasculature [86]. Therefore, comprehensive safety and biodistribution studies will be required before considering MK transfusion as an efficient alternative to PLT transfusion. However, this is a quickly evolving area where novel technical breakthroughs are expected to allow the sufficient supply of in vitro generated MKs and or PLTs to meet the high demands on transfusion and regenerative medicine.

\section{Disclosure Statement}

The authors declare no conflict of interest.

\section{References}

1 Golebiewska EM, Poole AW: Platelet secretion: from haemostasis to wound healing and beyond. Blood Rev 2015;29:153-162.

2 Semple JW, Italiano JE Jr, Freedman J: Platelets and the immune continuum. Nat Rev Immunol 2011;11: 264-274.

3 Klinger MH, Jelkmann W: Role of blood platelets in infection and inflammation. J Interferon Cytokine Res 2002;22:913-922.

4 Franco AT, Corken A, Ware J: Platelets at the interface of thrombosis, inflammation, and cancer. Blood 2015; 126:582-588.

5 Thon JN, Montalvo A, Patel-Hett S, Devine MT, Richardson JL, Ehrlicher A, Larson MK, Hoffmeister K, Hartwig JH, Italiano JE Jr: Cytoskeletal mechanics of proplatelet maturation and platelet release. J Cell Biol 2010;191:861-874.

6 Geddis AE: Megakaryopoiesis. Semin Hematol 2010; 47:212-219.

7 Pallotta I, Lovett M, Rice W, Kaplan DL, Balduini A Bone marrow osteoblastic niche: A new model to study physiological regulation of megakaryopoiesis. PLoS One 2009; 4:e8359.

8 Karagiannis P, Eto K: Manipulating megakaryocytes to manufacture platelets ex vivo. J Thromb Haemost 2015;13(suppl 1):S47-53.

9 Grozovsky R, Giannini S, Falet H, Hoffmeister KM: Regulating billions of blood platelets: glycans and beyond. Blood 2015;126:1877-1884.

10 Psaila B, Lyden D, Roberts I: Megakaryocytes, malignancy and bone marrow vascular niches. J Thromb Haemost 2012;10:177-188.

11 Kopp HG, Rafii S: Thrombopoietic cells and the bone marrow vascular niche. Ann N Y Acad Sci 2007;1106: 175-179.

12 Avecilla ST, Hattori K, Heissig B, Tejada R, Liao F Shido K, Jin DK, Dias S, Zhang F, Hartman TE, Hackett NR, Crystal RG, Witte L, Hicklin DJ, Bohlen P, Eaton D, Lyden D, de Sauvage F, Rafii S: Chemokinemediated interaction of hematopoietic progenitors with the bone marrow vascular niche is required for thrombopoiesis. Nat Med 2004;10:64-71.

13 Gars E, Rafii S: It takes 2 to thrombopoies in the vascular niche. Blood 2012;120:2775-2776.

14 Nishimura S, Nagasaki M, Kunishima S, Sawaguchi A, Sakata A, Sakaguchi H, Ohmori T, Manabe I, Italiano JE Jr, Ryu T, Takayama N, Komuro I, Kadowaki T, Eto $\mathrm{K}$, Nagai R: Il-1alpha induces thrombopoiesis through megakaryocyte rupture in response to acute platelet needs. J Cell Biol 2015;209:453-466.

15 Wang B, Zheng J: Platelet generation in vivo and in vitro. Springerplus 2016;5:787.

16 Sim X, Poncz M, Gadue P, French DL: Understanding platelet generation from megakaryocytes: implications for in vitro-derived platelets. Blood 2016;127:12271233.
17 Whitaker B, Rajbhandary S, Kleinman S, Harris A, Kamani N: Trends in United States blood collection and transfusion: results from the 2013 AABB blood collection, utilization, and patient blood management survey. Transfusion 2016;56:2173-2183.

18 Thon JN, Medvetz DA, Karlsson SM, Italiano JE Jr: Road blocks in making platelets for transfusion. J Thromb Haemost 2015;13(suppl 1):S55-62.

19 Kaufman RM, Djulbegovic B, Gernsheimer T, Kleinman S, Tinmouth AT, Capocelli KE, Cipolle MD, Cohn CS, Fung MK, Grossman BJ, Mintz PD, O'Malley BA, Sesok-Pizzini DA, Shander A, Stack GE, Webert KE, Weinstein R, Welch BG, Whitman GJ, Wong EC, Tobian AA; AABB: Platelet transfusion: a clinical practice guideline from the AABB. Ann Intern Med 2015;162:205-213.

20 Johnson B, Fletcher SJ, Morgan NV: Inherited thrombocytopenia: novel insights into megakaryocyte maturation, proplatelet formation and platelet lifespan. Platelets 2016;27:519-525.

21 Khodadi E, Asnafi AA, Shahrabi S, Shahjahani M, Saki $\mathrm{N}$ : Bone marrow niche in immune thrombocytopenia: a focus on megakaryopoiesis. Ann Hematol 2016;95: 1765-1776.

22 Thakkar RN, Lee KH, Ness PM, Wintermeyer TL, Johnson DJ, Liu E, Rajprasad A, Knight AM, Wachter PA, Demski R, Frank SM: Relative impact of a patient blood management program on utilization of all three major blood components. Transfusion 2016;56:2212-2220.

23 Sekhar M, Clark S, Atugonza R, Li A, Chaudhry Z: Effective implementation of a patient blood management programme for platelets. Transfus Med 2016;26:422431

24 Thomas S: Platelets: handle with care. Transfus Med 2016;26:330-338.

25 Handigund M, Cho YG: Insights into platelet storage and the need for multiple approaches. Ann Clin Lab Sci 2015;45:713-719.

26 Cho J: A paradigm shift in platelet transfusion therapy. Blood 2015;125:3523-3525.

27 Pavenski K, Rebulla P, Duquesnoy R, Saw CL, Slichter SJ, Tanael S, Shehata N; International Collaboration for Guideline Development, Implementation and Evaluation for Transfusion Therapies (ICTMG) CollaboratorsC: Efficacy of HLA-matched platelet transfusions for patients with hypoproliferative thrombocytopenia: a systematic review. Transfusion 2013;53:2230-2242.

28 Martinez CE, Smith PC, Palma Alvarado VA: The influence of platelet-derived products on angiogenesis and tissue repair: a concise update. Front Physiol 2015; 6:290.

29 Sarkar S, Alam MA, Shaw J, Dasgupta AK: Drug delivery using platelet cancer cell interaction. Pharm Res 2013;30:2785-2794.
30 Masoudi E, Ribas J, Kaushik G, Leijten J, Khademhosseini A: Platelet-rich blood derivatives for stem cellbased tissue engineering and regeneration. Curr Stem Cell Rep 2016;2:33-42.

31 Pezzotta S, Del Fante C, Scudeller L, Rossi GC, Perotti C, Bianchi PE, Antoniazzi E: Long-term safety and efficacy of autologous platelet lysate drops for treatment of ocular GvHD. Bone Marrow Transplant 2017;52: 101-106.

32 Ronci C, Ferraro AS, Lanti A, Missoroli F, Sinopoli S, Del Proposto G, Cipriani C, De Felici C, Ricci F, Ciotti M, Cudillo L, Arcese W, Adorno G: Platelet-rich plasma as treatment for persistent ocular epithelial defects. Transfus Apher Sci 2015;52:300-304

33 Burnouf T, Strunk D, Koh MB, Schallmoser K: Human platelet lysate: replacing fetal bovine serum as a gold standard for human cell propagation? Biomaterials 2016;76:371-387.

34 Bieback K: Platelet lysate as replacement for fetal bovine serum in mesenchymal stromal cell cultures. Transfus Med Hemother 2013;40:326-335.

35 Sun Y, Su J, Liu G, Chen J, Zhang X, Zhang R, Jiang M, Qiu M: Advances of blood cell-based drug delivery systems. Eur J Pharm Sci 2017;96:115-128.

36 Demers M, Wagner DD: Targeting platelet function to improve drug delivery. Oncoimmunology 2012;1:100102.

37 Kaushansky K: Historical review: Megakaryopoiesis and thrombopoiesis. Blood 2008;111:981-986.

38 Kuter DJ: Milestones in understanding platelet production: a historical overview. Br J Haematol 2014;165: 248-258.

39 Choi ES, Nichol JL, Hokom MM, Hornkohl AC, Hunt $P$ : Platelets generated in vitro from proplatelet-displaying human megakaryocytes are functional. Blood 1995; 85:402-413.

$40 \mathrm{Yu} \mathrm{M}$, Cantor AB: Megakaryopoiesis and thrombopoiesis: an update on cytokines and lineage surface markers. Methods Mol Biol 2012;788:291-303.

41 Lebois M, Dowling MR, Gangatirkar P, Hodgkin PD, Kile BT, Alexander WS, Josefsson EC: Regulation of platelet lifespan in the presence and absence of thrombopoietin signaling. J Thromb Haemost 2016;14:18821887.

42 Nurhayati RW, Ojima Y, Taya M: Recent developments in ex vivo platelet production. Cytotechnology 2016;68:2211-2221.

43 Minteer DM, Marra KG, Rubin JP: Adipose stem cells biology, safety, regulation, and regenerative potential. Clin Plast Surg 2015;42:169-179.

44 Kapur SK, Dos-Anjos Vilaboa S, Llull R, Katz AJ: Adipose tissue and stem/progenitor cells: discovery and development. Clin Plast Surg 2015;42:155-167. 
45 Ono-Uruga Y, Tozawa K, Horiuchi T, Murata M, Okamoto S, Ikeda Y, Suda T, Matsubara Y: Human adipose tissue-derived stromal cells can differentiate into megakaryocytes and platelets by secreting endogenous thrombopoietin. J Thromb Haemost 2016;14:12851297.

46 Siripin D, Kheolamai P, Y UP, Supokawej A, Wattanapanitch $M$, Klincumhom N, Laowtammathron C, Issaragrisil S: Transdifferentiation of erythroblasts to megakaryocytes using FLI1 and ERG transcription factors. Thromb Haemost 2015;114:593-602.

47 Ono Y, Wang Y, Suzuki H, Okamoto S, Ikeda Y, Murata $M$, Poncz $M$, Matsubara $Y$ : Induction of functional platelets from mouse and human fibroblasts by p45NF-E2/Maf. Blood 2012;120:3812-3821.

48 Pulecio J, Alejo-Valle O, Capellera-Garcia S, Vitaloni M, Rio P, Mejia-Ramirez E, Caserta I, Bueren JA, Flygare J, Raya A: Direct conversion of fibroblasts to megakaryocyte progenitors. Cell Rep 2016;17:671-683.

49 Nakamura S, Takayama N, Hirata S, Seo H, Endo H, Ochi K, Fujita K, Koike T, Harimoto K, Dohda T, Watanabe A, Okita K, Takahashi N, Sawaguchi A, Yamanaka S, Nakauchi H, Nishimura S, Eto K: Expandable megakaryocyte cell lines enable clinically applicable generation of platelets from human induced pluripotent stem cells. Cell Stem Cell 2014;14:535-548.

50 Schlinker AC, Radwanski K, Wegener C, Min K, Miller WM: Separation of in-vitro-derived megakaryocytes and platelets using spinning-membrane filtration. Biotechnol Bioeng 2015;112:788-800.

51 Miyazaki R, Ogata H, Iguchi T, Sogo S, Kushida T, Ito $\mathrm{T}$, Inaba M, Ikehara S, Kobayashi Y: Comparative analyses of megakaryocytes derived from cord blood and bone marrow. Br J Haematol 2000; 108:602-609.

52 Sun L, Tan P, Yap C, Hwang W, Koh LP, Lim CK, Aw SE: In vitro biological characteristics of human cord blood-derived megakaryocytes. Ann Acad Med Singapore 2004;33:570-575.

53 Thon JN, Italiano JE Jr: Does size matter in platelet production? Blood 2012;120:1552-1561.

54 Paniccia R, Priora R, Liotta AA, Abbate R: Platelet function tests: a comparative review. Vasc Health Risk Manag 2015;11:133-148.

55 Matsunaga T, Tanaka I, Kobune M, Kawano Y, Tanaka M, Kuribayashi K, Iyama S, Sato T, Sato Y, Takimoto R, Takayama T, Kato J, Ninomiya T, Hamada H, Niitsu Y: Ex vivo large-scale generation of human platelets from cord blood CD34+ cells. Stem Cells 2006;24: 2877-2887.

56 Mattia G, Vulcano F, Milazzo L, Barca A, Macioce G, Giampaolo A, Hassan HJ: Different ploidy levels of megakaryocytes generated from peripheral or cord blood CD34+ cells are correlated with different levels of platelet release. Blood 2002;99:888-897.

57 Ivetic N, Nazi I, Karim N, Clare R, Smith JW, Moore JC, Hope KJ, Kelton JG, Arnold DM: Producing megakaryocytes from a human peripheral blood source. Transfusion 2016;56:1066-1074.

58 Lu SJ, Li F, Yin H, Feng Q, Kimbrel EA, Hahm E, Thon JN, Wang W, Italiano JE, Cho J, Lanza R: Platelets generated from human embryonic stem cells are functional in vitro and in the microcirculation of living mice. Cell Res 2011;21:530-545.

59 Gaur M, Kamata T, Wang S, Moran B, Shattil SJ, Leavitt AD: Megakaryocytes derived from human embryonic stem cells: a genetically tractable system to study megakaryocytopoiesis and integrin function. J Thromb Haemost 2006;4:436-442.
60 Takayama N, Nishikii H, Usui J, Tsukui H, Sawaguchi A, Hiroyama T, Eto K, Nakauchi H: Generation of functional platelets from human embryonic stem cells in vitro via ES-SACS, VEGF-promoted structures that concentrate hematopoietic progenitors. Blood 2008; 111:5298-5306.

61 Feng Q, Shabrani N, Thon JN, Huo H, Thiel A, Machlus KR, Kim K, Brooks J, Li F, Luo C, Kimbrel EA, Wang J, Kim KS, Italiano J, Cho J, Lu SJ, Lanza R: Scalable generation of universal platelets from human induced pluripotent stem cells. Stem Cell Reports 2014;3: 817-831.

62 Liu Y, Wang Y, Gao Y, Forbes JA, Qayyum R, Becker L, Cheng L, Wang ZZ: Efficient generation of megakaryocytes from human induced pluripotent stem cells using food and drug administration-approved pharmacological reagents. Stem Cells Transl Med 2015;4: 309-319.

63 Borger AK, Eicke D, Wolf C, Gras C, Aufderbeck S, Schulze K, Engels L, Eiz-Vesper B, Schambach A, Guzman CA, Lachmann N, Moritz T, Martin U, Blasczyk R, Figueiredo C: Generation of HLA-universal iPSCsderived megakaryocytes and platelets for survival under refractoriness conditions. Mol Med 2016; doi: 10.2119/molmed.2015.00235.

64 Zhang N, Zhi H, Curtis BR, Rao S, Jobaliya C, Poncz M, French DL, Newman PJ: Crispr/cas9-mediated conversion of human platelet alloantigen allotypes. Blood 2016;127:675-680.

65 Moreau T, Evans AL, Vasquez L, Tijssen MR, Yan Y, Trotter MW, Howard D, Colzani M, Arumugam M, Wu WH, Dalby A, Lampela R, Bouet G, Hobbs CM, Pask DC, Payne H, Ponomaryov T, Brill A, Soranzo N, Ouwehand WH, Pedersen RA, Ghevaert C: Large-scale production of megakaryocytes from human pluripotent stem cells by chemically defined forward programming. Nat Commun 2016;7:11208.

66 Sullenbarger B, Bahng JH, Gruner R, Kotov N, Lasky LC: Prolonged continuous in vitro human platelet production using three-dimensional scaffolds. Exp Hematol 2009;37:101-110

67 Lasky LC, Sullenbarger B: Manipulation of oxygenation and flow-induced shear stress can increase the in vitro yield of platelets from cord blood. Tissue Eng Part C Methods 2011;17:1081-1088.

68 Pallotta I, Lovett M, Kaplan DL, Balduini A: Three-dimensional system for the in vitro study of megakaryocytes and functional platelet production using silkbased vascular tubes. Tissue Eng Part C Methods 2011; 17:1223-1232.

69 Di Buduo CA, Wray LS, Tozzi L, Malara A, Chen Y, Ghezzi CE, Smoot D, Sfara C, Antonelli A, Spedden E, Bruni G, Staii C, De Marco L, Magnani M, Kaplan DL, Balduini A: Programmable 3D silk bone marrow niche for platelet generation ex vivo and modeling of megakaryopoiesis pathologies. Blood 2015;125:2254-2264.

70 Nakagawa Y, Nakamura S, Nakajima M, Endo H, Dohda T, Takayama N, Nakauchi H, Arai F, Fukuda T, Eto K: Two differential flows in a bioreactor promoted platelet generation from human pluripotent stem cellderived megakaryocytes. Exp Hematol 2013;41:742748.

71 Thon JN, Mazutis L, Wu S, Sylman JL, Ehrlicher A, Machlus KR, Feng Q, Lu S, Lanza R, Neeves KB, Weitz DA, Italiano JE Jr: Platelet bioreactor-on-a-chip. Blood 2014; 124:1857-1867.

72 Thon JN, Dykstra BJ, Beaulieu LM: Platelet bioreactor: accelerated evolution of design and manufacture. Platelets 2017; doi: 10.1080/09537104.2016.
73 Blin A, Le Goff A, Magniez A, Poirault-Chassac S, Teste B, Sicot G, Nguyen KA, Hamdi FS, Reyssat M, Baruch D: Microfluidic model of the platelet-generating organ: beyond bone marrow biomimetics. Sci Rep 2016;6:21700.

74 Avanzi MP, Oluwadara OE, Cushing MM, Mitchell ML, Fischer S, Mitchell WB: A novel bioreactor and culture method drives high yields of platelets from stem cells. Transfusion 2016;56:170-178.

75 Guan X, Wang L, Zhang Y, Shen B, Ren Z, Ma Y, Dai W, Jiang Y: Pilot-scale production of megakaryocytes/ platelets from cord blood cd $34^{+}$cells in a bottle turning device culture system. Blood 2016;128:819.

76 Bertolini F, Battaglia M, Pedrazzoli P, Da Prada GA, Lanza A, Soligo D, Caneva L, Sarina B, Murphy S, Thomas T, della Cuna GR: Megakaryocytic progenitors can be generated ex vivo and safely administered to autologous peripheral blood progenitor cell transplant recipients. Blood 1997;89:2679-2688.

77 Xi J, Zhu H, Liu D, Nan X, Zheng W, Liu K, Shi W, Chen L, Lv Y, Yan F, Li Y, Xie X, Wang Y, Yue W, Xu $X$, Wei X, Zhu J, Huang X, Pei X: Infusion of megakaryocytic progenitor products generated from cord blood hematopoietic stem/progenitor cells: results of the phase 1 study. PLoS One 2013;8:e54941.

78 https://clinicaltrials.gov/ct2/show/NCT02241031?term= megakaryocyte↔ $r a n k=2$.

79 https://upload.umin.ac.jp/cgi-open-bin/icdr_e/ctr_view. cgi? recptno $=$ R000017851.

80 Heazlewood SY, Nilsson SK, Cartledge K, Be CL, Vinson A, Gel M, Haylock DN: Progress in bio-manufacture of platelets for transfusion. Platelets 2017; doi: 10.1080/09537104.2016.1257783.

81 Balduini A, Di Buduo CA, Kaplan DL: Translational approaches to functional platelet production ex vivo. Thromb Haemost 2016;115:250-256.

82 Bakchoul T, Fuhrmann J, Chong BH, Bougie D, Aster R, Subcommittee on Platelet Immunology: Recommendations for the use of the non-obese diabetic/severe combined immunodeficiency mouse model in autoimmune and drug-induced thrombocytopenia: communication from the SSC of the ISTH. J Thromb Haemost 2015;13:872-875.

83 Newman PJ, Aster R, Boylan B: Human platelets circulating in mice: applications for interrogating platelet function and survival, the efficacy of antiplatelet therapeutics, and the molecular basis of platelet immunological disorders. J Thromb Haemost 2007;5(suppl 1):305-309.

84 Jirouskova M, Shet AS, Johnson GJ: A guide to murine platelet structure, function, assays, and genetic alterations. J Thromb Haemost 2007;5:661-669.

85 Smith BW, Murphy GJ: Stem cells, megakaryocytes, and platelets. Curr Opin Hematol 2014;21:430-437.

86 Fuentes R, Wang Y, Hirsch J, Wang C, Rauova L, Worthen GS, Kowalska MA, Poncz M: Infusion of mature megakaryocytes into mice yields functional platelets. J Clin Invest 2010;120:3917-3922.

87 Engert A, Balduini C, Brand A, Coiffier B, Cordonnier C, Dohner H, de Wit TD, Eichinger S, Fibbe W, Green T, de Haas F, Iolascon A, Jaffredo T, Rodeghiero F, Salles G, Schuringa JJ; EHA Roadmap for European Hematology Research: The European Hematology Association Roadmap for European Hematology Research: a consensus document. Haematologica 2016; 101:115-208. 